\title{
Optimal Channel Selection for Simultaneous RF Energy Harvesting and Data Transmission in Cognitive Radio Networks
}

\author{
A. Hooshiary ${ }^{1}$, P. Azmi ${ }^{*}$, N. Mokari ${ }^{1}$, S. Maleki² \\ ${ }^{1}$ Department of Electrical and Computer Engineering, Tarbiat Modares University, Tehran, Iran. \\ 2 Iran Interdisciplinary Center for Security and Reliability, University of Luxembourg, Luxembourg.
}

\begin{abstract}
In this paper, an RF-powered cognitive radio network is considered, in which the secondary users are powered by an RF energy harvester (Rectenna). Unlike most existing works, we consider a realistic Rectenna characteristic function, and derive the actual amount of harvested energy and thus, the resulting actual energy level of the secondary users. We consider a system architecture at which simultaneous energy harvesting and data transmission for each secondary user is possible. We introduce a strategy to manage the challenge of network throughput decreasing due to lack of the secondary users' energy, via selecting the best possible channels for energy harvesting and simultaneously by allocating the best channels for data transmission. Therefore, we implement cognition in spectrum utilization and in energy harvesting. We show that the amount of harvested energy affects the available energy of the secondary user and consequently the throughput, therefore, the channels selection to maximize energy harvesting affects the network throughput. To maximize the network throughput, the Hungarian algorithm is employed, and then, an algorithm with lower complexity based on the matching theory is proposed. Finally, we compare our proposed approach with some existing benchmarks and show its high performance in energy harvesting and system throughput.
\end{abstract}

${ }^{*}$ Correspondence

P. Azmi, Department of Electrical and Computer Engineering, Tarbiat Modares University, Tehran, Iran.

E-mail: pazmi@modares.ac.ir

\section{INTRODUCTION}

Energy harvesting is considered as a promising solution for extending the lifetime of communication networks by introducing self-sustainability for energy-limited devices. Energy can be harvested from different sources including solar, radio frequency (RF), wind, thermal gradients, mechanical vibrations, etc [1,2].The ambient RF power is a good potential candidate for the energy supply as it is widely broadcasted from numerous reliable electromagnetic resources. Unlike most of the energy sources, the $\mathrm{RF}$ energy sources do not depend on the nature, therefore, they are more controllable at the receivers compared to conventional natural energy sources [3,4]. However, since the power density of the ambient RF sources is small, it is very challenging to design RF energy harvesting systems with a high RF-to-DC power conversion efficiency. The key element of an RF energy harvesting system is a device called Rectenna (rectifying antenna) [5], which converts the received RF power to DC power.
Cognitive radio, which allows opportunistic spectrum sharing between primary (licensed) users and secondary (unlicensed) users to improve spectral efficiency, is considered as one of the main technologies for solving the wireless spectrum scarcity problem.

Therefore, the energy harvesting cognitive radio network (EHCRN) is emerged to satisfy two wireless communication requirements, i.e., increasing energy efficiency and spectral efficiency. Energy efficiency of EHCRN enhances due to its harvesting capability, that may lead to perpetually operation without need for a wired external power supply [3]. At the same time, EHCRN also improves spectral efficiency by dynamically accessing the underutilized licensed spectrum.

In an EHCRN that is equipped with the RF energy harvesting device, to obtain enough energy and spectrum opportunity for data transmission, the secondary user (SU) should search for not only an idle channel of primary users (PUs) to transmit its data, but also should search for a busy channel to harvest RF energy. Such a contradictory necessities, makes throughput optimization in RF powered 
EHCRNs an interesting problem to solve.

It should be noted that in order to maximize the throughput in EHCRNs where the SUs harvest energy, the main factors are energy level of the SUs and channel access opportunities. Therefore, proper channel selection for energy harvesting and proper channel selection for data transmission are the key elements which affect the throughput of such networks.

\subsection{Related Work}

There are some research works studying different issues of RF energy harvesting cognitive wireless network [6,7]. A policy for deciding between harvesting and transmitting, to achieve an optimal balance between the immediate throughput and the harvested energy for future use, is proposed in [3].

In [8], a Markov decision process is formulated to obtain an optimal channel selection policy to maximize the longterm average throughput of the SU for a multiple-channel RF-powered EHCRN.

The channel selection problem is considered in $[9,10]$. In [9], the authors have formulated a Markov decision process model and used an offline method to find an optimal policy to maximize the average throughput of the SU in the case where the SU has incomplete information about the channels. In [10], an online method is developed that allows finding an optimal solution to maximize average throughput of the $\mathrm{SU}$ when the $\mathrm{SU}$ has no information about the channels. In [11], the authors proposed a decentralized channel selection policy for EHCRN, where the SUs can either harvest RF energy from PUs' transmission or obtain channel access for data transmission. In [8]-[10], each SU selects a channel for energy harvesting and also estimates the resulting energy level regardless of the realistic parameters of the energy harvester and only based on statistical information e.g., probability of successful packet transmission, probability of a packet arrival at the SU, probability of successful RF energy harvesting and idle probability of channels. In [3], [8]-[11], the SU transmits its data with a fixed power. In [3], [6] and [8]-[11], the SU selects a channel, if the selected channel is idle the SU can transmit its data and if the selected channel is occupied the SU can harvest energy from the PU's received RF power.

Nevertheless, to the best of our knowledge, the actual energy level of the SUs through implementation of Rectenna conversion efficiency has not been investigated in the existing literature. A novel contribution of the present paper is to obtain the actual harvested energy of the SU by considering the characteristic function of Rectenna and consequently calculate the resulting energy level.

\subsection{Contributions}

In this paper, we consider an EHCRN, where the SUs harvest energy from PUs' received RF power. The main contributions of this paper are summarized as follows:
- Unlike existing works, which calculate the energy level of the SUs only based on statistical information and without taking into account the energy harvester realistic parameters, we derive formula for calculating the actual value of SUs' harvested energy by considering the realistic parameters of Rectenna and also considering the network parameters. It is worth noting that nonlinearity of Rectenna conversion efficiency is also considered in the derived formula.

- We manage the challenge of the required energy in the RF-powered EHCRNs, by simultaneously selecting the best possible channels for energy harvesting as well as allocating the best channel for data transmission. Through the proposed method, we minimize the system throughput reduction due to the shortage of the SUs' energy.

- The performance of proposed method is compared with other existing approaches and shown that the proposed method performs better than the existing ones.

- We present the problem of channel selection for maximizing energy harvesting and the problem of channel allocation for maximizing the network throughput as two binary linear programming (BLP) problems. Then, for maximizing the expected achievable throughput, we employ the Hungarian algorithm and the matching theory algorithm. The proposed matching theory algorithm has lower complexity compared to the Hungarian algorithm. Its performance is lower but close to the Hungarian algorithm.

The rest of this paper is organized as follows:

Section 2 presents a review on Rectenna. Section 3 describes the system model and assumptions of this paper. In Section 4, we discuss channel selection methods and the underlying optimization formulas and also, we derive solutions for optimization problems. The simulation results are given in Section 5. Finally, the paper is concluded in Section 6.

\section{REVIEW OF RECTENNA}

The goal of an ambient RF energy harvester is to convert the RF energy received from ambient RF sources into DC power. Fig. 1 shows an RF energy harvester, composed of an RF antenna, an impedance matching, a voltage multiplier and a capacitor to collect the RF signals and convert them into DC power. The antenna can be designed to work on either single or multiple frequency bands. In other words, the Rf energy harvester can harvest from a single or multiple sources simultaneously [6]. The impedance matching is a resonator circuit operating at the designed frequency to maximize the power transfer 


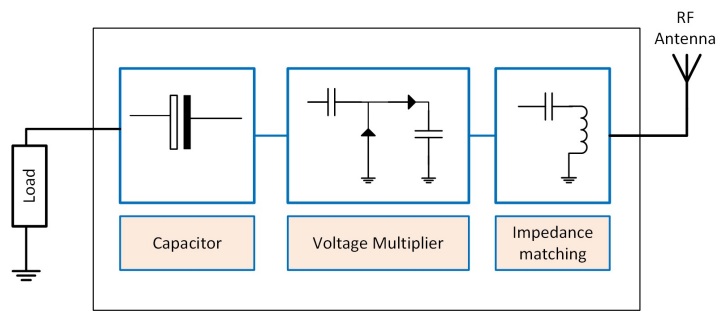

Figure 1. General structure of an RF energy harvester [6].

between the antenna and the voltage multiplier. The main components of the voltage multiplier are diodes of the rectifying circuit which convert RF signals (AC signals in ambient) into DC voltage. The capacitor ensures to deliver power smoothly to the load. Additionally, when RF energy is unavailable, the capacitor can also serve as a reserve for a short duration [6].

The RF to DC conversion efficiency of the RF energy harvester is equal to the ratio of DC output power to the input ambient RF power. It should be noted that the Rectenna characteristic function is nonlinear [12], therefore, the optimal channel selection for energy harvesting considering the Rectenna characteristic functions is a critical issue. If this is not taken into account, the result of system analysis may differ significantly from the actual behavior of the network. We consider this issue in the problem formulations of this paper.

\section{SYSTEM MODEL}

We consider an RF-powered EHCRN, that consists multiple PUs (M) and multiple SUs (N). The PUs utilize their channels to transmit data on a time slot basis. All PUs follow the same time slot structure. In each time slot, a channel can either be idle (free) or occupied (busy).

Here, it is considered that the SUs are capable to harvest RF energy from $K$ frequency bands of PUs and $K \leq$ $M$. An schematic channels selection model for optimal energy harvesting and optimal data transmission is shown in Fig. 2. The harvested energy from PU's channel depends on the active status of the PU. The activity status of the PU is assumed to be a Bernoulli process [13].

The traffic of primary network is modeled as a timehomogeneous discrete Markov process, in which the spectrum randomly switches its states between idle and occupied according to a discrete Markov process [14, 15]. The spectrum of the $m^{\text {th }}$ frequency band transits from idle state to occupied state with probability $1-P_{1 m}$ and stays in occupied state with probability $1-P_{0 m}$. Using Markov chain analysis, the steady-state probabilities of being idle and occupied for the $m^{\text {th }}$ primary channel are given by $\pi_{i m}$ and $\pi_{o m}$, respectively as follows [15]:

$\pi_{i m}=\frac{1-P_{0 m}}{2-P_{1 m}-P_{0 m}} \quad$ and $\quad \pi_{o m}=\frac{1-P_{1 m}}{2-P_{1 m}-P_{0 m}}$.

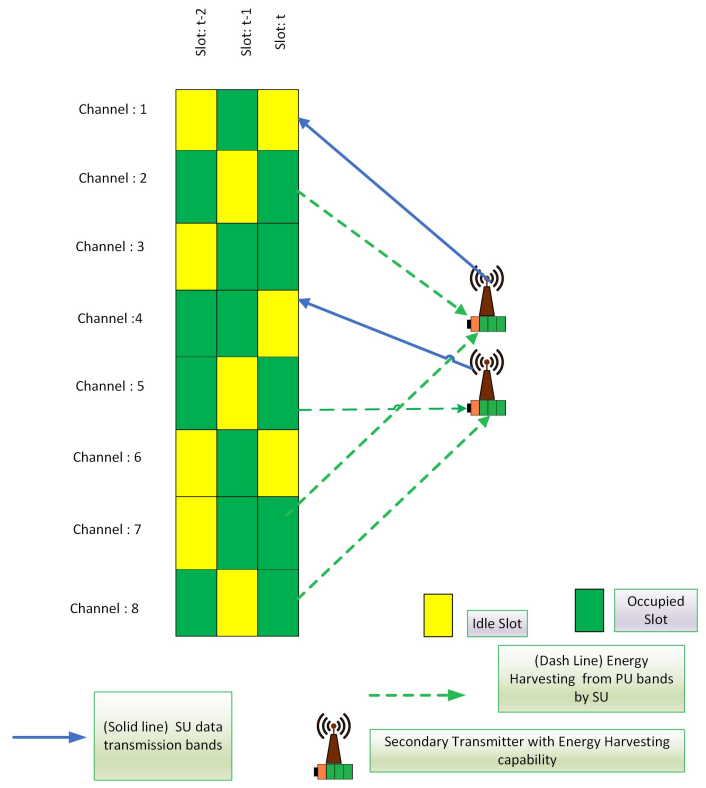

Figure 2. Schematic model for channels selection for optimal energy harvesting and data transmission, $\mathrm{K}=2$

It is assumed that the transition probabilities, $P_{0 m}$ and $P_{1 m}$ are known to the EHCRN through long-term spectrum measurements. We use the energy detector method because it does not require any information about the PUs' signal, and also it is easy to implement and has the low computational complexity.

If we consider $f_{l m}$ and $f_{h m}$ as the lowest and highest marginal frequencies of the band $f_{m}$, when it is mentioned that Rectenna can harvest from frequency band $f_{m}$, this actually means that it can harvest from $f_{l m}$ to $f_{h m}$.

Another assumption of this paper is that the SUs are equipped with separate RF energy harvester and transceiver. Therefore, the SUs can perform energy harvesting and data transmitting simultaneously. This system is applicable for EHCRNs with RF energy harvesting, and practically it is useful for low rate cognitive sensor networks which are compatible with standards such as the IEEE 802.5.14.

\section{OPTIMAL CHANNELS SELECTIONS FOR ENERGY HARVESTING AND DATA TRANSMISSION}

In this section, we formulate the underlying problems and provide solutions to maximize the throughput of the systems. It consists of two main stages; the purpose of the first stage is to select the best channels such that the energy level of the SUs is maximized. In the second stage, according to the energy level that was obtained from the first stage, the best channel is assigned to each SU such that the network throughput is maximized. Therefore, the 
goal of our proposed scheme is to maximize the harvested energy level and maximize the network throughput, briefly we call it "MEHMT" (maximize energy harvesting \& maximize throughput).

\subsection{Channel Selection for Energy Harvesting}

In EHCRNs which the SUs harvest energy and they are not fed by a fixed power supply, maintaining their energy level is crucial. This is because in the case of lack of energy, their data transmission breaks, and in turn, no throughput can be expected. Here, we devise an approach which can be utilized to calculate the actual value of SUs' harvested energy and their resulting energy level.

The channel between the $m^{t h}$ primary transmitter and the $n^{t h} \mathrm{SU}$ is denoted by $h_{m n}$ and $h_{m n}=g_{m n} d_{m n}^{-\delta}$, where $g_{m n}$ is exponential random variable representing the Rayleigh fading, $d_{m n}$ is distance between the $m^{t h}$ PU's transmitter and the $n^{t h}$ SU's receiver and $\delta$ is path loss exponent. The received RF power at the $n^{\text {th }} \mathrm{SU}$ from the $m^{t h}$ primary transmitter is $P_{R, m n}=P_{T m}\left|h_{m n}\right|^{2}$, where $P_{T m}$ is the transmission power of the $m^{t h}$ PU [16].

The output voltage of Rectenna for the $n^{t h}$ SU in the case of harvesting energy from the $m^{\text {th }}$ frequency band, is derived as follows [17]:

$$
V_{\text {out }, m n}=\frac{1}{2}\left(\left|V_{r f, m n}\right|-\frac{n V_{T}}{2} \ln \left(2 \pi \frac{\left|V_{r f, m n}\right|}{n V_{T}}\right)\right),
$$

where $n$ and $V_{T}$ denote ideality factor and thermal voltage for diode of Rectenna, respectively. $V_{r f, m n}$ presets the input voltage of the Rectenna for the $n^{\text {th }} \mathrm{SU}$ when it harvests from the $m^{t h}$ frequency band.

The output voltage of Rectenna for the $n^{\text {th }}$ SU when it harvests from $M$ frequency bands, is obtained by [17]:

$$
V_{\text {out }, M n}=\frac{1}{2} \sum_{m=1}^{M}\left(\left|V_{r f, m n}\right|-\frac{n V_{T}}{2} \ln \left(2 \pi \frac{\left|V_{r f, m n}\right|}{n V_{T}}\right)\right) .
$$

Lemma 1. The output power of Rectenna for the $n^{\text {th }} S U$, when it harvests from $m^{\text {th }}$ frequency band is obtained as follows:

$$
\begin{aligned}
& P_{\text {out }, m n}=\frac{\left(V_{\text {out }, m n}\right)^{2}}{R_{L}}=\frac{1}{4 R_{L}}\left\{\left(2 P_{T m}\left|h_{m n}\right|^{2} R_{a, n}\right)^{\frac{1}{2}}-\right. \\
& \left.\frac{n V_{T}}{2} \ln \left(2 \pi \frac{\left(2 P_{T m}\left|h_{m n}\right|^{2} R_{a, n}\right)^{\frac{1}{2}}}{n V_{t}}\right)\right\}
\end{aligned}
$$

Proof. Receiving antenna of the $n^{\text {th }}$ SU is modeled as a simple $R_{a, n}$ resistor at the selected frequencies. As in general, $P=\frac{\left(V_{r m s}\right)^{2}}{R}$ and $V_{r m s}=\frac{V}{\sqrt{2}}$, where $P$ denotes power and $V_{r m s}$ is root mean square voltage, then $V=$ $(2 P R)^{\frac{1}{2}}$, hence the input voltage of Rectenna for the $n^{t h}$ SU when it harvests from the $m^{t h}$ frequency band, is as follows:

$$
V_{r f, m n}=\left(2 P_{R, m n} R_{a, n}\right)^{\frac{1}{2}} .
$$

By substituting $P_{R, m n}$ in (5), we obtain

$$
V_{r f, m n}=\left(2 P_{T m}\left|h_{m n}\right|^{2} R_{a, n}\right)^{\frac{1}{2}}
$$

By combining (2) and (6), the output power of Rectenna is derived as (4), where $R_{L}$ denotes the load resistance. By considering Lemma 1 and (3), the output power of Rectenna for the $n^{t h} \mathrm{SU}$ when it harvests from $M$ frequency bands, is obtained as follows:

$$
\begin{aligned}
& P_{\text {out }, M n}=\frac{\left(V_{\text {out }, M n}\right)^{2}}{R_{L}}=\frac{1}{4 R_{L}} \sum_{m=1}^{M}\left\{\left(2 P_{T m}\left|h_{m n}\right|^{2}\right.\right. \\
& \left.\left.R_{a, n}\right)^{\frac{1}{2}}-\frac{n V_{T}}{2} \ln \left(2 \pi \frac{\left(2 P_{T m}\left|h_{m n}\right|^{2} R_{a, n}\right)^{\frac{1}{2}}}{n V_{t}}\right)\right\}
\end{aligned}
$$

The harvested energy by the $n^{t h}$ SU from the $m^{t h}$ PU's channel is obtained as follows:

$$
\begin{aligned}
& e_{h, m n}=\frac{T-\tau}{4 R_{L}}\left\{\left(2 P_{T m}\left|h_{m n}\right|^{2} R_{a, n}\right)^{\frac{1}{2}}-\frac{n V_{T}}{2}\right. \\
& \left.\ln \left(2 \pi \frac{\left(2 P_{T m}\left|h_{m n}\right|^{2} R_{a, n}\right)^{\frac{1}{2}}}{n V_{T}}\right)\right\},
\end{aligned}
$$

where $\tau$ and $T$ denote the sensing time and slot duration, respectively. In (8), uncertainty in the channel status identification is not considered. Thus, it is required to implement the imperfect sensing parameters including detection and false alarm probabilities. The SU can harvest energy from primary channels in two states:

- The primary channel is reported as busy and actually is busy with the probability $\left(1-\pi_{i m}\right) P_{d, m n}$.

- The primary channel is reported as idle, but actually is busy, this means there is a false alarm, the probability for such cases to occur is $\pi_{i m} P_{f, m n}$.

Therefore, the average harvested energy is obtained as follows:

$$
\begin{aligned}
& E_{h, m n}=\frac{T-\tau}{4 R_{L}}\left\{\left(2 P_{T m}\left|h_{m n}\right|^{2} R_{a, n}\right)^{\frac{1}{2}}-\frac{n V_{T}}{2} \ln (2 \pi\right. \\
& \left.\left.\frac{\left(2 P_{T m}\left|h_{m n}\right|^{2} R_{a, n}\right)^{\frac{1}{2}}}{n V_{T}}\right)\right\}\left\{\left(1-\pi_{i m}\right) P_{d, m n}+\pi_{i m} P_{f, m n}\right\},
\end{aligned}
$$

where $\pi_{i m}$ and $\pi_{o m}$ denote the idle and occupied status of the $m^{t h}$ primary channel, respectively i.e.,

$$
1-\pi_{i m}=\pi_{o m}=\left\{\begin{array}{ll}
1 & , \text { occupied status } \\
0 & , \text { idle status }
\end{array} .\right.
$$

Also, $P_{d, m n}$ denotes the detection probability of the $m^{t h}$ PU's band by the $n^{t h} \mathrm{SU}$ and $P_{f, m n}$ is the false alarm probability of the $m^{\text {th }}$ PU's band by the $n^{\text {th }}$ SU [18]. $P_{d, m n}$ and $P_{f, m n}$ are obtained as follows:

$$
\begin{gathered}
P_{d, m n}=Q\left(\left(\frac{\varepsilon}{\sigma_{n}^{2}}-\gamma_{m n}-1\right) \sqrt{\tau f_{s}}\right) \\
P_{f, m n}=Q\left(\left(\frac{\varepsilon}{\sigma_{n}^{2}}-1\right) \sqrt{\tau f_{s}}\right)
\end{gathered}
$$

where $Q=\int_{x}^{\infty} \exp \left(-\frac{u^{2}}{2}\right) \mathrm{d} u$ is the complementary distribution function of the standard Gaussian distribution, 
$\gamma_{m n}$ is the received signal to noise ratio (SNR) of the $m^{\text {th }}$ band measured at the $n^{\text {th }} \mathrm{SU}, \varepsilon$ denotes the detection threshold for all the SUs, $f_{s}$ is the sampling frequency of the received signal from PU for the $n^{\text {th }} \mathrm{SU}, \tau$ is a multiple of $\frac{1}{f_{s}}$, and thus the number of samples is $\tau f_{s}$.

The considered optimization problem for optimal channel selection to harvest energy is derived as follows:

$$
\begin{array}{cl}
\max _{\rho} & \sum_{m=1}^{M} \sum_{n=1}^{N} \rho_{m n} E_{h, m n}, \\
\text { s.t. : } & \sum_{m=1}^{M} \rho_{m n}=\mathrm{K}, \forall n, \\
& \forall \rho_{m n} \in\{0,1\},
\end{array}
$$

where $\rho_{m n}$ is the assignment index and is set to " 1 " if the $n^{t h}$ SU selects the channel of the $m^{t h}$ PU, otherwise it is set to " 0 ". The constraint (12b) denotes that each SU is capable to harvest from $K$ primary channels.

The optimization problem is a BLP problem, as its objective function and constraints can be decoupled into $N$ sub-problems, therefore, to solve problem (12), it can be transformed into $N$ sub-problems. Howevere, to maximize energy harvesting, each SU $n$ selects $K$ channels which provide the highest harvesting energy.

\subsection{Channel Allocation for Data Transmission}

Here, our goal is to maximize the total throughput of the SUs by considering the results of the energy harvesting. We presented the channel selection problem for maximizing the harvested energy in Section 4.1. The throughput of the $n^{t h} \mathrm{SU}$ when it operates in the absence of the $m^{t h} \mathrm{PU}$ is denoted by $C_{0, m n}$, and $C_{1, m n}$ denotes the throughput of the $n^{t h} \mathrm{SU}$ when it operates in the presence of the $m^{t h}$ PU. Considering the case in which there is only one pointto-point transmission in the secondary network, the SNR for this secondary link is denoted by $S N R_{s}$, and $S N R_{p}$ denotes the received SNR from the PU at the SU's receiver. Then $C_{0, m n}=\log _{2}\left(1+S N R_{s}\right)=$ $\log _{2}\left(1+P_{t n} \gamma_{s n}\right)$ and $C_{1, m n}=\log _{2}\left(1+\frac{S N R_{s}}{1+S N R_{p}}\right)=$ $\log _{2}\left(\frac{P_{t n} \gamma_{s n}}{1+S N R_{p}}\right)$, where $\gamma_{s n}$ denotes the channel to noise ratio of the $n^{t h}$ SU. $P_{t n}$ denotes the transmission power of the $n^{t h} \mathrm{SU}$ and $P_{t n}=x P_{t n, a}$, where $P_{t n, a}$ denotes the maximum available power for transmission of the $n^{\text {th }} \mathrm{SU}$ and $x$ is a coefficient to define the utilized portion of the available power for transmission of the SU. Also $P_{t n, a}$ is defined as follows:

$$
P_{t n, a}=(T-\tau) E_{n}(t),
$$

where $E_{n}(t)$ is the energy level of the $n^{t h} \mathrm{SU}$ at the beginning of time slot $t$ and is expressed as follows:

$$
\begin{aligned}
& E_{n}(t)=E_{n}(t-1)+E_{h, m n}(t-1)-1_{x} E_{c n}(t-1)- \\
& 1_{y} E_{s}(t-1)
\end{aligned}
$$

where $E_{n}(t-1)$ denotes the amount of energy remaining in the battery of the $n^{\text {th }} \mathrm{SU}$ at the beginning of time slot $t-1$, also $E_{h, m n}(t-1)$ is the amount of the $n^{t h} \mathrm{SU}$ 's harvested energy from the $m^{t h}$ primary channels during slot $t-1, E_{c n}(t-1)$ is the consumed energy for data transmission of the $n^{t h} \mathrm{SU}$ during slot $t-1, E_{s}(t-1)$ is consumed energy for spectrum sensing in slot $t-1$ and $E_{n}(0)=0$. Indicator function $1_{x}$, is equal to one if $x$ is true, otherwise is equal to zero. On the other hand, $x$ is true if data transmission is performed. Also $1_{y}$ is an indicator function, $y$ is true if the SU has enough energy to transmit data. It is worth noting that the energy consumption for spectrum sensing of all the SUs is assumed to be of the same value.

To utilize PU's frequency band by the SU there are the following two scenarios:

- Scenario I: When the $m^{t h}$ PU is not present and the $n^{t h}$ SU produces no false alarm, then the achievable throughput of the $n^{t h} \mathrm{SU}$ is $\frac{T-\tau}{T} C_{0, m n}$. The probability of occurring this condition is $(1-$ $\left.P_{f, m n}\right) \pi_{i, m}$. Therefore, the average throughput for this case is as follows:

$$
R_{0, m n}=\frac{T-\tau}{T} C_{0, m n}\left(1-P_{f, m n}\right) \pi_{i, m}
$$

- Scenario II: When the $m^{t h} \mathrm{PU}$ is active but the $n^{t h}$ SU does not detect it, then the achievable throughput of the $n^{t h} \mathrm{SU}$ is $\frac{T-\tau}{T} C_{1, m n}$. The probability of occurring this condition is $\left(1-P_{d, m n}\right)\left(1-\pi_{i, m}\right)$. Therefore, the average throughput for this scenario is as follows:

$$
R_{1, m n}=\frac{T-\tau}{T} C_{1, m n}\left(1-P_{d, m n}\right)\left(1-\pi_{i, m}\right) .
$$

Therefore, when the $n^{t h}$ SU utilizes the $m^{\text {th }}$ channel of PU for data transmission, the average throughput is calculated as follows:

$$
R_{m n}=R_{0, m n}+R_{1, m n} .
$$

The higher detection probability protects a PU's transmission from the interferences caused by the SU's transmission. Thus, the constraint $P_{d} \geq \beta$ is defined as a PU protection, where $\beta$ is a threshold for acceptable detection probability. Since in practice the considered threshold for $P_{d, m n}$ is very close to 1 [18], $\left(1-P_{d, m n}\right)$ is always a very small value. Lower false alarm probability provides the opportunity of using idle channel for the SUs and in practice the false alarm probability is lower than 0.1 [19]. Since $C_{0, m n} \geq C_{1, m n}$ [18] and considering the above explanation, $R_{0, m n}$ dominates the achievable throughput. In other words, by proper spectrum sensing, the throughput of the SU when it operates in presence of the PU is negligible. Among primary channels, only those channels can be allocated to a SU that their detection probability by that $\mathrm{SU}$ is desirable, i.e., $\mathcal{C}_{m n}=\left\{m ; P_{d, m n} \geq \beta\right\}$.

As we described, only the SUs that have enough energy can 
transmit their data, thus we define a set of the SUs denoted by $\mathcal{S}_{n}$, such that $\mathcal{S}_{n}=\left\{n ; E_{n} \geq E_{t h}\right\}$, where $E_{t h}$ is the target energy that is required for the SUs data transmission. Optimization problem in order to allocate the best channels to SUs for data transmission and to maximize the related total throughput is cast as follows:

$$
\begin{aligned}
\max _{\varphi} & \sum_{n \in \mathcal{S}_{n}} \sum_{m \in \mathcal{C}_{m n}} \varphi_{m n} R_{m n}, \forall m \in \mathcal{C}_{m n}, \forall n \in \mathcal{S}_{n} \\
& \mathcal{S}_{n}=\left\{n ; E_{n} \geq E_{t h}\right\} ; \mathcal{C}_{m n}=\left\{m ; P_{d, m n} \geq \beta\right\} \\
\text { s.t. : } & \sum_{m=1}^{M} \varphi_{m n} \leq 1 \quad \forall n \in \mathcal{S}_{n}, \\
& \sum_{n=1}^{N} \varphi_{m n} \leq 1 \quad \forall m \in \mathcal{C}_{m n}, \\
& \forall \varphi_{m n} \in\{0,1\},
\end{aligned}
$$

where $\varphi_{m n}$ is assignment index and is set to " 1 " if the $m^{\text {th }}$ primary channel is allocated to the $n^{\text {th }} \mathrm{SU}$ for data transmission, otherwise it is set to " 0 ". The constraint (18b) assures that at most one primary channel is assigned to one SU, whereas (18c) ensures that one channel should be selected just for one SU.

Considering the aforementioned explanation, we observe that $E_{h, m n}$ which is obtained from (12), has effect in calculation of $E_{n}(t)$, on the other hand, transmission power of the SU is related to $E_{n}(t)$, i.e., $P_{t n}=x(T-$ $\tau) E_{n}(t)$, therefore, the throughput maximization problem depends on the energy harvesting maximization problem. The optimization problem is again a BLP problem. We utilize the Hungarian algorithm to solve it optimally in polynomial time, as the complexity of this algorithm is high, we also propose a lower complexity method based on the matching theory algorithm and later we show that our proposed algorithm performs near to the optimal solution.

\subsubsection{Matching-Based Channel Allocation}

In order to obtain a less complex solution for the channel allocation formulation and to solve the optimization problem, we employ the matching theory algorithm as the mathematical framework to form relationships between primary channels and SUs. Matching theory approach has the following advantages to solve the presented optimization problem (18): 1) as our model is decentralized, matching theory can be applied properly, 2) matching theory algorithm allows SUs and primary channels to define their individual utilities based on their local information, 3) efficient algorithmic implementations that are inherently self-organizing and amenable to fast implementation, 4) low complexity and acceptable optimality [20]. This technique divides the matching players into two distinct sets and each member of a set ranks a subset of other set's members in the order of preference. The preference of one set over the other set is derived from the local information available to

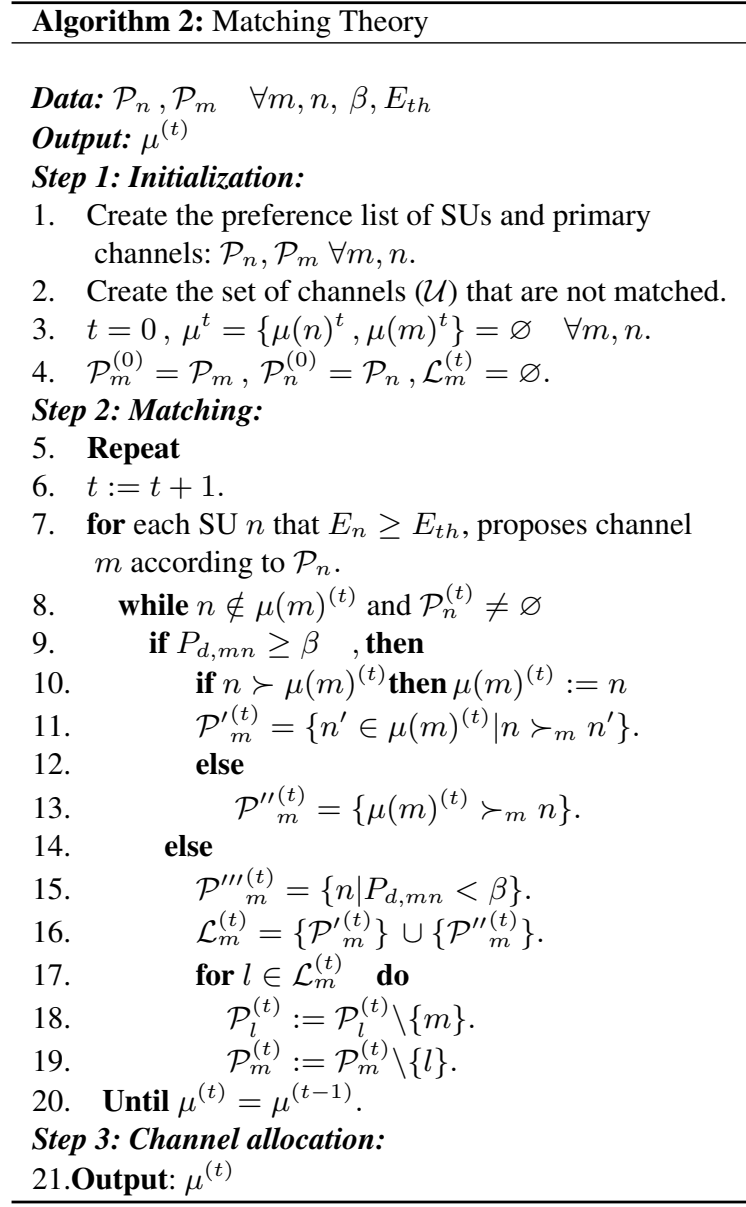

each member. The channel allocation matching problem is defined in order to find the best matching between SUs and primary channels. Based on the constraints (18b) and (18c), our design corresponds to a one-to-one matching given by the related sets $\left(\mathcal{M}, \mathcal{N}, \succ_{\mathcal{M}}, \succ_{\mathcal{N}}\right)$. Here $\succ_{\mathcal{M}} \triangleq\left\{\succ_{m}\right\}_{m \in \mathcal{M}}$ and $\succ_{\mathcal{N}} \triangleq\left\{\succ_{n}\right\}_{n \in \mathcal{N}}$ represent the set of the preference relations of the SUs and primary channels, respectively.

Definition 1: A matching $\mu$ is defined by a function from the set $\mathcal{M} \cup \mathcal{N}$ into the set of elements of $\mathcal{M} \cup \mathcal{N}$ such that

1. $|\mu(n)| \leq 1$ and $\mu(n) \in \mathcal{M}$,

2. $|\mu(m)| \leq 1$ and $\mu(m) \in \mathcal{N} \cup \varnothing$,

3. $\mu(n)=m$ if and only if $n$ is in $\mu(m)$,

where $\mu(n)=\{m\} \Leftrightarrow \mu(m)=\{n\}$ for $\forall m \in \mathcal{M}$ and $\forall n \in \mathcal{N}$, also $|\mu()$.$| denotes the cardinality of matching$ outcome $\mu($.$) . The first two conditions state that the$ matching is a one-to-one relation such that a primary channel $m$ can be allocated to only one SU (18b), and cannot be shared among different SUs (18c). Note that $\mu(m)=\varnothing$, when a SU $n$ is not allowed to use a primary channel $m$. 


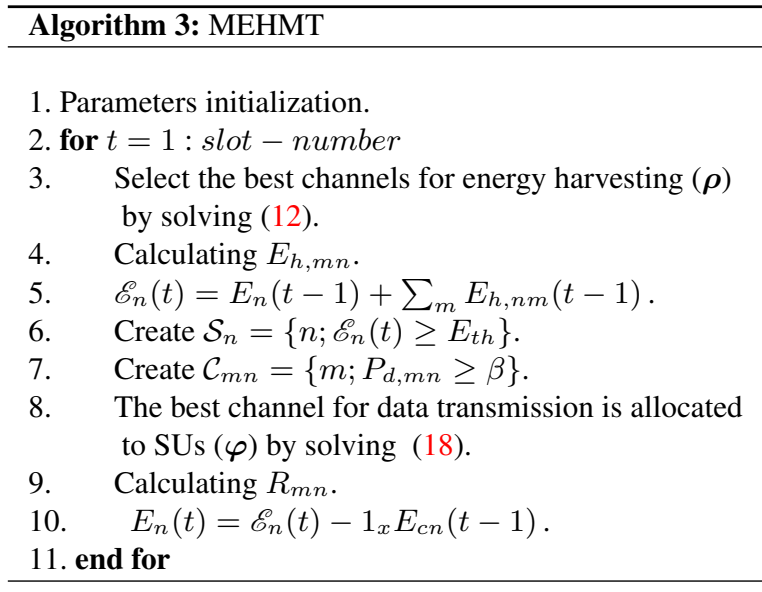

\subsubsection{Preferences List}

Matching is performed in accordance to preference profiles which are formed by the SUs and primary channels to rank potential matchings with considering the local information. Note that each SU $n$ chooses primary channel $m$ based on the following preference function

$$
\mathcal{U}_{n}=R_{m n}
$$

which is obtained from the objective of our problem, i.e., throughput maximization of each SU. Therefore, each SU ranks all the primary channels $m \in \mathcal{M}$ in non-increasing order in its preference list which is introduced by $\mathcal{P}_{n}$. Similarly, each primary channel also requires to has a preference list of the SUs $\left(\mathcal{P}_{m}\right)$ which are ranked in a non-increasing order, and is provided by its following preference function

$$
\mathcal{U}_{m}=P_{d, m n}-\beta
$$

\subsubsection{Proposed Matching Algorithm}

In this section, we present the allocation algorithm based on the matching approach and the purpose of this algorithm is to find a stable allocation which is a main solution concept in matching theory [21,22]. A matching $\mu$ is stable if no other pair $(m, n)$ exists, where $m \in \mathcal{M}$ and $n \in \mathcal{N}$, such that $m \succ_{n} \mu(n)$ and $n \succ_{m} \mu(m)$ where $\mu(n)$ and $\mu(m)$ represent the current matched partners of $n$ and $m$, respectively.

The pseudo code is given in Algorithm 2. There are three steps for performing matching process. In the first step, all SUs start the initialization process by computing their utilities regarding primary channels and also primary channels perform such calculation regarding the SUs. Then, the non-increasing order preference list of all primary channels and SUs are created (i.e., $\mathcal{P}_{m}, \mathcal{P}_{n}$ ). A set of unmatched primary channels are created as $\mathcal{U}$. At the beginning of the algorithm, all the primary channels
Table I. Complexity and Performance Comparison

\begin{tabular}{|c|c|c|}
\hline Number of PUs \& SUs & C & P \\
\hline$M=8, N=5$ & $16 \%$ & $12 \%$ \\
\hline$M=8, N=6$ & $40 \%$ & $14 \%$ \\
\hline$M=8, N=7$ & $55 \%$ & $18 \%$ \\
\hline$M=6, N=5$ & $40 \%$ & $16 \%$ \\
\hline
\end{tabular}

$\mathrm{C}=$ Difference between complexity of Hungarian and matching algorithms.

$\mathrm{P}=$ Difference between performance of Hungarian and matching algorithms.

Table II. Channel selection methods in the presented approaches

\begin{tabular}{|c|c|c|}
\hline $\begin{array}{c}\text { Algorithm } \\
\text { Name }\end{array}$ & $\begin{array}{c}\text { Channel Selection } \\
\text { Method for Energy } \\
\text { Harvesting }\end{array}$ & $\begin{array}{c}\text { Solution Method for } \\
\text { Maximizing } \\
\text { Throughput Problem }\end{array}$ \\
\hline $\begin{array}{c}\text { MEHMT- } \\
\text { MT }\end{array}$ & problem (12) & Matching theory \\
\hline $\begin{array}{c}\text { MEHMT- } \\
\text { HU }\end{array}$ & problem (12) & Hungarian \\
\hline MD & $\begin{array}{c}\text { SU selects } K \\
\text { nearest primary } \\
\text { channels }\end{array}$ & Hungarian \\
\hline RA & $\begin{array}{c}\text { SU selects } K \\
\text { channels } \\
\text { randomly }\end{array}$ & Hungarian \\
\hline HoT & problem (12) & Hungarian \\
\hline
\end{tabular}

are in the $\mathcal{U}$. In the second step, each SU $n$ which has sufficient energy for data transmission but no primary channel has been assigned to it, based on its preference list proposes to its most preferred primary channel $m$. Then, the primary channel first determines the detection probability $\left(P_{d, m n}\right)$ relevant to the SU $n$, if $P_{d, m n}$ is below the predefined detection probability threshold, that primary channel is added to the rejected list. If not rejected, then the primary channel checks the preference ranking based on $\mathcal{P}_{m}$ for the $\mathrm{SU} n$, if $\mathrm{SU} n$ has a higher preference utility than the current match $\left(\mu(m)^{t}\right)$, the current match $n^{\prime}$ will be rejected and the SU $n$ will be matched to the $m$. Moreover, the rejected SU $n^{\prime}$ will be added in the rejected list $\mathcal{P}_{m}^{\prime(t)}$. Also, if the SU $n$ has a lower preference than the current match $\left(\mu(m)^{t}\right), n$ will be rejected and added in the rejected list $\mathcal{P}_{m}^{\prime \prime}{ }_{m}^{(t)}$. Finally, at iteration $t$ all the rejected SUs, i.e., the set $\mathcal{L}_{m}^{(t)}$, is then used by SUs and primary channels to update their preference lists. Thus, each primary channel $m$ removes the rejected SU $n$ from the $\mathcal{P}_{m}^{(t)}$, and also these SUs remove $m$ from $\mathcal{P}_{n}^{(t)}$.

Iteration of matching process is continued until a stable 


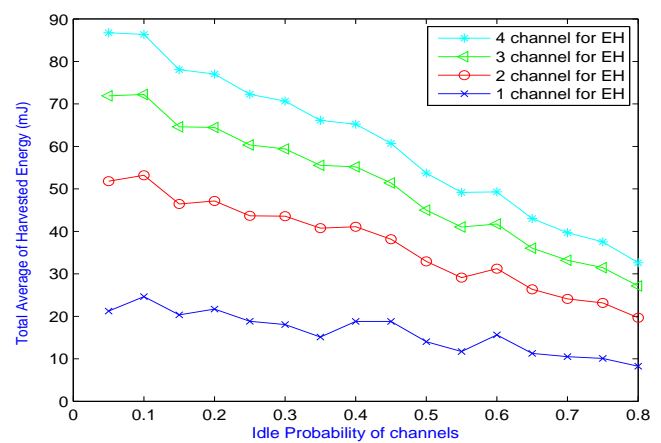

(a) Total average of harvested energy

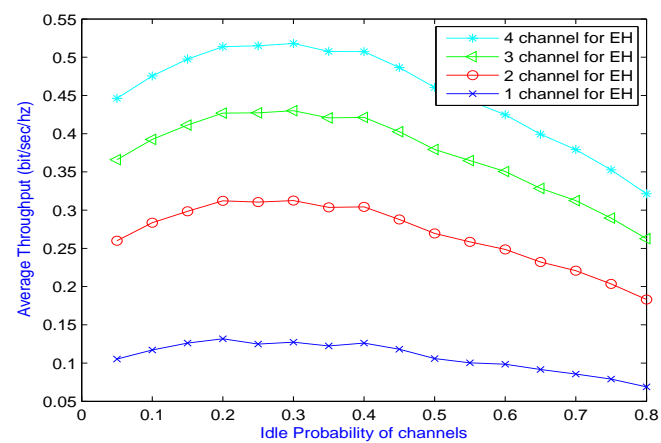

(b) Average Throughput

Figure 3. Total average of harvested energy and throughput of the SUs versus idle probability of primary channels for different $K$.

match is found between both sides. When a primary channel is allocated to a SU which have enough energy, and the matching of two consecutive iterations remains unchanged, the algorithm converges and the process is stopped. Finally, based on the result of the mentioned process, the matched SUs are allowed to transmit their data on the matched allocated channels.

\subsubsection{Complexity}

In the proposed matching theory algorithm, the SUs and primary channels in initialization phase should construct their preference list using standard sorting algorithm (e.g. merge sort, heap sort [23]), and thus the complexity is $\mathcal{O}(N M \log (N M))$. In the matching phase, each $\mathrm{SU}$ proposes the most preferred channel, hence the number of iteration at most is $N$. On the other hand in each iteration, at most $M$ channels are assigned, thus the complexity is $\mathcal{O}(M N)$. Therefore, the total complexity of the proposed algorithm is $\mathcal{O}(N M \log (N M))+\mathcal{O}(N M)$. It worth noting that the delay time of the matching theory algorithm by increasing the number of PUs and SUs does not increase exponentially. For instance, the elapsed time for running the algorithm for the case $M=8$ and $N=2$ is 0.223 millisecond, while for $M=10$ and $N=8$ the elapsed time is 0.976 millisecond.

\subsubsection{Comparing the Matching Theory with the Hungarian Algorithm}

We utilize the Hungarian algorithm to solve the throughput optimization problem (18) optimally with polynomial time. The complexity of this algorithm is $O\left(N^{3}\right)[24,25]$. We also propose a lower complexity method based on the matching theory algorithm and we show that our proposed algorithm is close to the optimal. Differences between complexity and performance of the Hungarian as well as the proposed matching theory algorithm are presented in Table I.

We can see that the complexity of the proposed matching algorithm is lower than that of the Hungarian algorithm. As an example for $M=8$ and $N=6$, the complexity of the proposed matching theory algorithm is $40 \%$ lower than the Hungarian algorithm, while for this case its performance is only $14 \%$ lower than the performance of the Hungarian algorithm.

As already mentioned, the throughput of the proposed system depends on energy levels of SUs, because in the cases that the energy level is less than minimum required energy, no data can be transmitted, and thus no throughput can be excepted for that SU. The proposed algorithm (MEHMT) is summarized in Algorithm 3. As already explained the purpose of this algorithm is selecting the best channel for energy harvesting and simultaneously allocating the best channel for data transmission to maximize network throughput.

\section{SIMULATION RESULT}

In this section, a brief description of algorithms and parameters setting are described. Also analysis of result under various system parameters are presented.

\subsection{Brief Description of Algorithms}

We present the MEHMT approach to maximize the harvested energy and the network throughput simultaneously. We present other approaches, which we call them, minimum distance (MD) and random (RA) algorithms. Also we utilize an existing algorithm [26] which we call it harvesting or transmitting (HoT). We compared these algorithms with each other. It should be noted that in all of the aforementioned algorithms, the amount of harvested energy is calculated based on (9). We considered two structures for energy harvesting and data transmission i.e., 'MEHMT' and 'HoT'. In MEHMT energy harvesting and data transmission are performed simultaneously, but in 


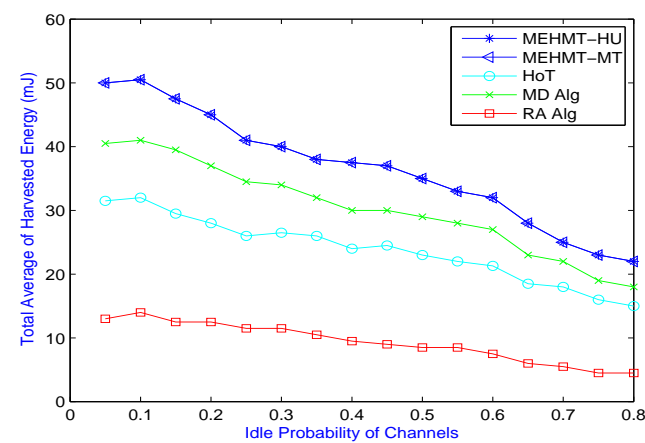

(a) Total average of harvested energy

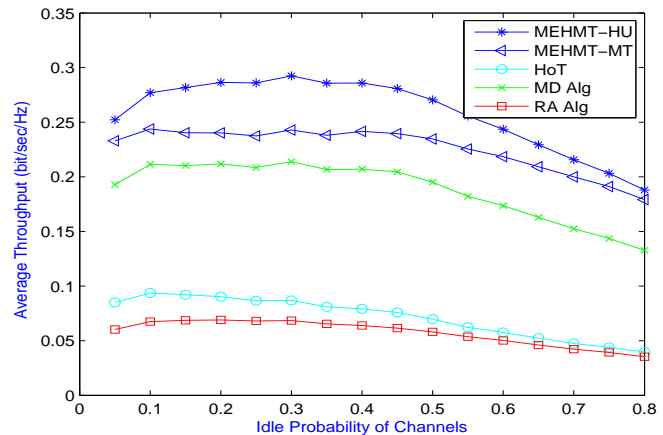

(b) Average Throughput

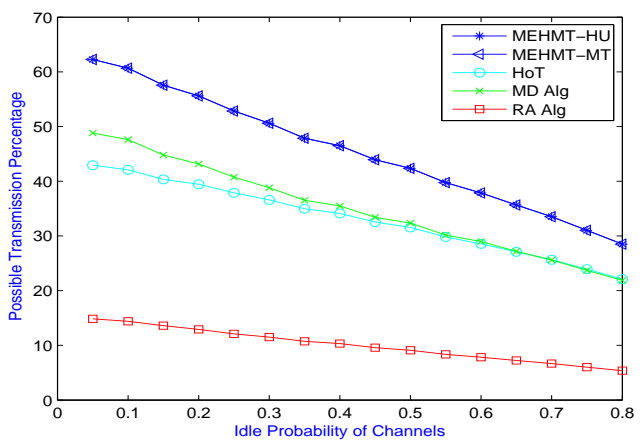

(c) Possible transmission percentage

Figure 4. Total average of harvested energy, throughput and possible transmission percentage of the SUs under different idle probability of primary channels, $K=2$.

HoT either harvesting or transmitting is performed. In HoT, if the SU has enough energy, it transmits its data and doesn't harvest energy, but if it doesn't have enough energy it harvests energy and cannot transmit its data. For MEHMT, we investigate four channel selection approaches to harvest energy and compared them with each other and also with HoT. A brief description about these approaches is presented in Table II.

\subsection{Parameters Setting}

Here we consider an RF-powered EHCRN which is distributed in an area of $500 \times 500 \mathrm{~m}^{2}$, five SUs and eight PUs are randomly located. It is assumed that GSM900, GSM1800, UMTS2100 and UTMS1900 are utilized by SUs for harvesting energy and data transmission. The GSM900 entire band is from 876 to $959 \mathrm{MHz}$, the GSM1800 is from 1710 to $1880 \mathrm{MHz}$, the UMTS2100 band is from 1920 to $2170 \mathrm{MHz}$ and finally the UTMS1900 band is from 1850 to $1990 \mathrm{MHz}$. The SUs are capable to harvest from $K$ frequency bands, here for $K=1,2,3,4$ results are presented. The ideality factor, the thermal voltage of Rectenna, the load and the antenna resistance, are drawn from [17], and are set to 1.3, 23 millivolts, 11 $\mathrm{k} \Omega$ and $50 \Omega$, respectively.

Target detection probability of PUs' band $(\beta)$ is set to 0.9 . The target of energy that is required for data transmission $\left(E_{t h}\right)$ of the SUs is set to $0.6 \mathrm{~mJ}$ [17].

Also, to validate the performance of the maximum energy harvesting and maximum throughput approach (MEHMT), we perform simulation for 50 time slots. The slot duration, sensing time and required energy for spectrum sensing are set to $T=0.1 \mathrm{sec}, \tau=1 \mathrm{~ms}$ and $E_{s}=0.1 \mathrm{~mJ}$, respectively.

\subsection{Analysis on Results}

Fig. 3a and Fig. 3b show the total average of harvested energy and the average throughput of the SUs versus the idle probability of channels, for different numbers of $K$, respectively. As illustrated, by increasing the number of channels that the SUs are capable to harvest from them, the total average of harvested energy and the throughput of the SUs increase.

Fig. 4a and Fig. 4b show the total average of harvested energy and throughput of the SUs versus the idle probability of channels, respectively. As the idle probability of channels increases (i.e. becomes less 


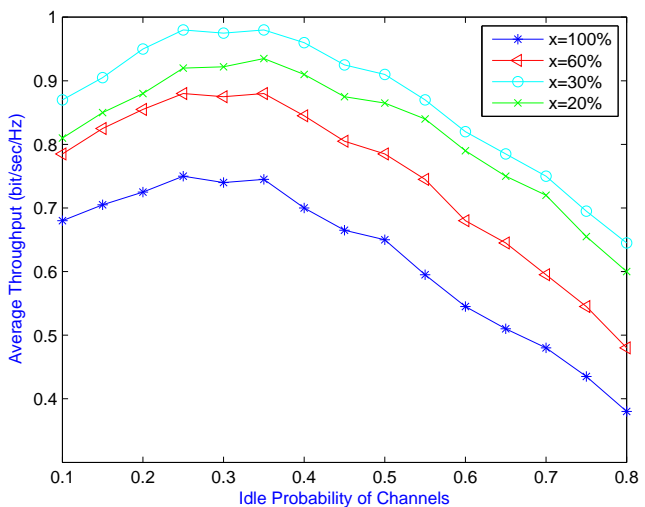

Figure 5. Average Throughput, $M=6, N=2, K=4$

occupied), the total average of harvested energy decreases (Fig. 4a), because the SUs have less chance to harvest energy. As shown in Fig. 4b, by increasing the idle probability of channels, the throughput first increases and then, decreases. The increment is because, the SUs have more chance to transmit their data and still have enough energy. On the other hand, when the channels are mostly idle, the SUs cannot harvest much RF energy, therefore, they dont have enough energy to transmit their data, thus the throughput decreases.

As shown in Fig. 4a, the total average of harvested energy for MEHMT-MT and MEHMT-HU approaches are the same and the highest, the lowest is RA policy. Although the HoT approach either harvests or transmits its data, but here HoT harvested energy is superior than the RA approach and is near to the MD approach, because HoT selects the best channels for energy harvesting while RA selects the channels randomly. As shown in Fig. 4b, MEHMT-HU and MEHMT-MT approaches have the highest throughput, MEHMT-HU is the optimal solution and its throughput is slightly higher, but its complexity is higher too. Disadvantage of MEHMT-HU is high complexity, but our proposed matching theory algorithm (MEHMT-MT) has lower complexity and is near to optimal solution. Since HoT either harvests or transmits, loses some opportunities, therefore, the throughput of MD is higher than that of HoT. Regarding the RA, as it selects the channels for energy harvesting randomly its energy level is the lowest,and consequently its throughput is lowest too. Fig. 4c shows possible transmission percentage (PTP) of the approaches when the idle probability of channels is varied. The MEHM-MT and the MEHM-HU have the highest PTPs, and the lowest is for RA approach. PTPs of MD and HoT approaches are near to each other. Generally, for all approaches by increasing the idle probability of channels due to decrement of harvested energy, PTP decreases.

Fig. 5 shows the average throughput of the SUs versus idle probability for different power transmissions of the SUs. Unlike conventional wireless networks, which have no
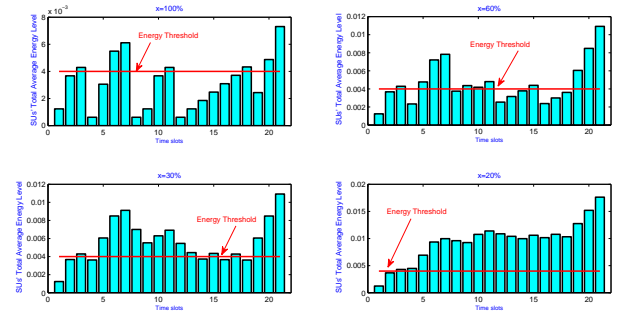

Figure 6. Total average energy level of the SU, $M=6, N=2$, $K=4$
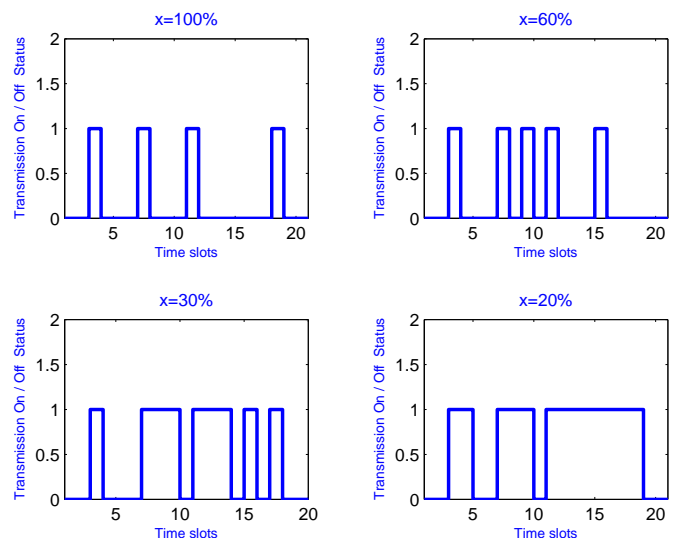

Figure 7. Transmission on/off status, $M=6, N=2, K=4$

limitation in the power supply, for our presented EHCRN system, we can see that by increasing the transmission power of the SUs, the throughput may not be increased. For instance, in Fig. 5, when the transmission powers of the SUs are set to $30 \%$ of their available power, the highest throughput is obtained. The reason is that when the transmission powers of the SUs are higher (i.e., $x=60 \%$ or $\mathrm{x}=100 \%$ ), in more slots the SUs confront with lack of the remaining energy and only waiting for energy harvesting, without any data transmission. This interval results in missing the available spectrum opportunities and leads to a reduction in the average throughput of the SUs. When the SUs transmission power are set to lower value than that of the optimal value (e.g., 20\%), although they transmit in more time slots, due to reduction of their transmit power, the related average throughput is lower and the energy is not utilized optimally. For better explaining about reason of this matter, the total average energy level of the SUs and the transmission on/off status in some time slots are illustrated in Fig. 6 and Fig. 7, respectively. For instance, in Fig. 6, in time slot 11, the remaining energy level of the SUs for $x=100 \%$ and $x=30 \%$ is higher than required energy for transmission $\left(E_{t h}\right)$, therefore, in both cases the SUs were able to transmit. After these transmissions for $x=100 \%$ until time slot 17 no further transmission is performed, but for $x=30 \%$ in this interval (from time slot 11 until 17) in four time slots transmission is performed. However, the network throughput depends on both the 
number of transmissions and the value of transmission power.

\section{CONCLUSION}

We considered an RF-powered EHCRN that is consist of multiple SUs and multiple primary channels. By utilizing the nonlinear characteristic function of the Rectenna, we derived formulas for calculating the actual harvested energy of the SUs from the primary channels and their resulting energy level. The amount of harvested energy affects the available energy of the SUs and consequently their throughput, therefore, by selecting the best possible channels for energy harvesting and simultaneously allocating the best channel for data transmission, we proposed an approach to manage the challenge of required energy such that the maximum harvested energy and maximum throughput are obtained. It is shown that the proposed approach significantly achieves higher throughput compared to the previous existing works. We also investigated the effects of harvested energy on the system throughput, we observed that as the idle probability of channels increases, the throughput first increases and then, decreases. The increment is because, the SUs have more chance to transmit their data and still have enough energy. On the other hand, when channels are mostly idle, the SUs cannot harvest much RF energy, therefore, they don't have enough energy to transmit their data, thus the throughput decreases.

Also it is shown that unlike conventional wireless networks which have no limitation in the power supply, for our presented EHCRN system, by increasing the transmission power of the SUs, due to lack of the SUs' remaining energy, the throughput may not be increased. As the extension to this work, some other traffic models can be considered and also other types of EHCRN paradigm such as underlay schemes can be investigated.

\section{REFERENCES}

1. LU X, Wang P, Niyato D and Kim DI. Wireless Charging Technologies: Fundamentals, Standards, and Network Applications. IEEE Communications Surveys \& Tutorials; Nov. 2016, 18, (2), pp. 14131452.

2. Alvarado U, Juanicorena A, Adin I, Sedano B, Gutierrez I and de No J. Energy harvesting technologies for low-power electronics. Transactions on Emerging Telecommunications Technologies; May. 2012, 23, pp. 728-741.

3. Park S, Heo J and Kim B. Optimal Mode Selection for Cognitive Radio Sensor Networks with RF Energy Harvesting. In Proceedings. IEEE PIMRC; Sept. 2012, pp. 2155-2159.
4. Lu X, Wang P and Niyato D. Resource Allocation in Wireless Networks with RF Energy Harvesting and Transfer. IEEE Network; Dec. 2015, 29, (6), pp. 6875.

5. Akkermans JAG, van Beurden MC and Doodeman GJN. Analytical Models for Low-Power Rectenna Design. IEEE Antennas and Wireless Propagation Letters; Jun. 2005, 4, pp. 187-190.

6. Lu X, Wang P and Niyato D. Wireless Networks with RF Energy Harvesting, A Contemporary Survey. IEEE Communications Surveys \& Tutorials; May. 2015, 19, (2), pp. 757-789.

7. Park S, Kim $\mathrm{H}$ and Hong D. Cognitive Radio Networks with Energy Harvesting. IEEE Wireless Communication; Mar. 2013, 12, (3), pp. 1386-1397.

8. Lu X, Wang D and Hossain E. Dynamic Spectrum Access in Cognitive Radio Networks with RF Energy Harvesting. IEEE Wireless Communication; Jun. 2014, 21, (3), pp. 102-110.

9. Niyato D, Wang P and Kim DI. Channel Selection in Cognitive Radio Networks with Opportunistic RF Energy Harvesting. In Proceedings. IEEE ICC; Sydney, Australia, Jun. 2014, pp.1555-1560.

10. Hoang DT, Niyato D and Wang P. Opportunistic Channel Access and RF Energy Harvesting in Cognitive Radio networks. IEEE Journal on Selected Areas in Communications; Nov. 2014, 32, (11), pp. 2039-2052, 2039-2052.

11. Pratibha, Li KH and Teh KC. Channel Selection in Multichannel Cognitive Radio Systems Employing RF Energy Harvesting. IEEE Transactions on Vehicular Technology; Jan. 2016, 65, (1), pp. 457-462.

12. Song C, Huang $\mathrm{Y}$ and Zhang J. A High-Efficiency Broadband Rectenna for Ambient Wireless Energy Harvesting. IEEE Transactions on Antennas and Propagation; Aug. 2015, 63, (8), pp. 3486-3495.

13. Papoulis A and Pillai S. Random Variables and Stochastic Processes. New York: McGraw-Hill, 4 ed, 2002.

14. Zhao Q, Tong L and Swami A. Decentralized Cognitive Mac for Opportunistic Spectrum Access in Ad Hoc Networks: A POMDP Framework. IEEE Journal on Selected Areas in Communications; Apr. 2007, 25, (3), pp. 589-600.

15. Chen Y, Zhao Q and Swami A. Joint Design and Separation Principle for Opportunistic Spectrum Access in the Presence of Sensing Errors. IEEE Transactions on Information Theory; May. 2008, 54, (5), pp. 2053-2071.

16. Goldsmith A. Wireless Communications. Cambridge, United Kingdom: Cambridge University Press, 2005.

17. Kuhn V, Lahuec C and Seguin F. A MultiBand Stacked RF Energy Harvester with RF-toDC Efficiency Up to $84 \%$ '. IEEE Transactions on Microwave Theory and Techniques; May. 2015, 63, (5), pp. 1768-1778. 
18. Liang YC, Zeng Y and Peh ECY. Sensing-Throughput Tradeoff for Cognitive Radio Networks. IEEE Transaction Wireless Communication; Apr. 2008, 7, (4), pp. 1326-1337.

19. Atapattu S., Tellambura C., Jiang H. Conventional Energy Detector. In: Energy Detection for Spectrum Sensing in Cognitive Radio. SpringerBriefs in Computer Science. Springer, New York, NY, 2014.

20. Gu Y, Saad W and Bennis M. Matching Theory for Future Wireless Networks: Fundamentals and Applications. IEEE Communications Magazine; May. 2015, 53, (5), pp. 52-59.

21. Roth AE. Deferred Acceptance Algorithms: History, Theory, Practice, and Open Questions. International Journal of Game Theory; Mar. 2008, 36, (3), pp.537569.

22. Gale D and Shapley L. College Admissions and the Stability of Marriage. Mathematical Association of America; Jan. 1962, 69, (1), pp. 9-15.

23. Shi $\mathrm{H}$ and Schaeffer J. Parallel Sorting by Regular Sampling. Journal of Parallel and Distributed Computing; Apr. 1992, 14, (14), pp. 361-372.

24. D. B. West, Introduction to Graph Theory. Prentice Hall, 2001.

25. Ma B, Shah-Mansouri H, and Wong V. W. S. A Matching Approach for Power Efficient Relay Selection in Full Duplex D2D Networks. In Proceedings. IEEE ICC, Kuala Lumpur, Malaysia, May. 2016, pp. 1-6.

26. Chung W, Park S and Lim S. Optimal Transmit Power Control for Energy-Harvesting Cognitive Radio System. In Vehicular Technology Conference (VTC Fall); Sep. 2013, pp. 1-5. 